\title{
Parallel Packing Squares into a Triangle
}

\author{
Janusz Januszewski, Xi Liu, Zhanjun Su, and Łukasz Zielonka®
}

\begin{abstract}
Assume that $T_{h}$ is a triangle with the interior angles at the base of the measure not greater than $90^{\circ}$, with the base length 1 and the height $h$. Let $S$ be a square with a side parallel to the base of $T_{h}$ and let $\left\{S_{n}\right\}$ be a collection of the homothetic copies of $S$. A tight upper bound of the sum of the areas of squares from $\left\{S_{n}\right\}$ that can be parallel packed into a triangle $T_{h}$ is determined.
\end{abstract}

Mathematics Subject Classification. 52C15, 05B40.

Keywords. Packing, parallel packing, square, triangle.

\section{Introduction}

Let $P$ be a polygon and let $S_{i}$ be a square for $i=1,2, \ldots$ One side of $P$ is called the base of $P$. A collection $S_{1}, S_{2}, \ldots$ is said to be packed into $P$ if their union is contained in $P$ and if these squares have pairwise disjoint interiors. A packing is called parallel if a side of each packed square is parallel to the base of $P$.

The goal is to pack the squares into $P$ with high density. The area of a polygon $R$ is denoted by $|R|$. Let $\varrho(P)$ be the greatest number such that any collection of squares of the total area not greater than $\varrho(P) \cdot|P|$ can be parallel packed into $P$.

Assume that $S$ is a square and that $T_{h}$ is a triangle with the interior angles at the base of the measure not greater than $90^{\circ}$, with the base length 1 and the height $h$. There are many results concerning packing squares or rectangles.

This research was partially supported by National Natural Science Foundation of China (11471095), the NSF of Hebei Province (A2021205008), and the Science Foundation of Hebei Normal University (L2020Z01). 

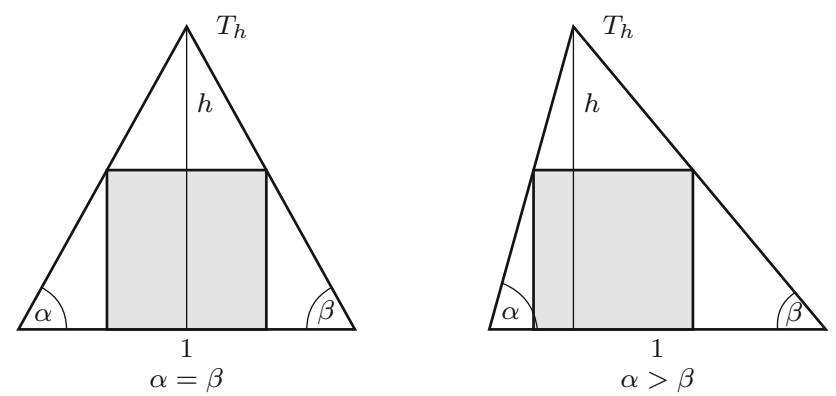

Figure 1. One square in $T_{h}$

Moon and Moser [11] showed that $\varrho(S)=1 / 2$; in this case the most effective usual packing is the parallel packing. In many publications in which squares are placed into a rectangle, when authors write "packing" they mean "parallel packing" (see for example [1,2,5,8-10,12]). Let us add that for packing squares or rectangles of the side lengths not greater than 1 into a large square the most effective packings are not parallel (see $[3,7]$ ).

The following results concerning parallel packing squares into a triangle were known:

- $\varrho\left(T_{\sqrt{3} / 2}\right)=2 \sqrt{3}-3$ for an equilateral triangle $T_{\sqrt{3} / 2}$ (see [6]),

- $\varrho\left(T_{\sqrt{2}}\right) \geq(16-6 \sqrt{2}) / 23$ for a right triangle $T_{\sqrt{2}}$ (see [4]),

- $\varrho\left(T_{\sqrt{2} / 2}\right)=6 \sqrt{2}-8$ and $\varrho\left(T_{\sqrt{2} / 3}\right)=6 \sqrt{2} /(11+6 \sqrt{2}) \quad($ see $[13])$.

The aim of this note is to show that

$$
\varrho\left(T_{h}\right)=\min \left\{\frac{2 h}{(h+1)^{2}}, \max \left[\frac{4 h}{(2 h+1)^{2}}, \frac{4 h}{(h+2)^{2}}\right]\right\} .
$$

Without loss of generality one can assume that $\alpha \geq \beta$ (see Fig. 1).

\section{Upper Bounds}

Claim 1. A square of the side length not greater than $h /(h+1)$ can be parallel packed into $T_{h}$ while one square of the side length greater than $h /(h+1)$ cannot.

Proof. Let $a$ be the greatest number such that the square of the side length equal to a can be parallel packed into $T_{h}$ (see Fig. 1). By Thales's intercept theorem, $\frac{1}{a}=\frac{h}{h-a}$, i.e., $a=\frac{h}{h+1}$.

Corollary 1. Any square of the area not greater than $2 h /(h+1)^{2} \cdot\left|T_{h}\right|$ can be parallel packed into $T_{h}$. Moreover, $\varrho\left(T_{h}\right) \leq 2 h /(h+1)^{2}$.

Proof. If the area of a square equals $2 h /(h+1)^{2} \cdot\left|T_{h}\right|=h^{2} /(h+1)^{2}$, then its side length is equal to $h /(h+1)$. 


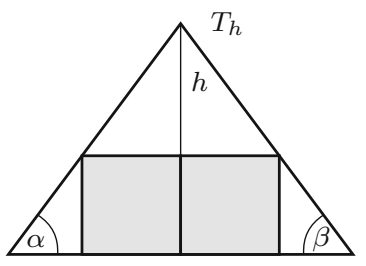

$\alpha=\beta$

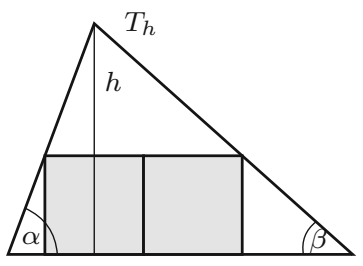

$\alpha>\beta$

Figure 2. Two congruent squares in $T_{h}$ for $\sqrt{2} / 2<h \leq 1$
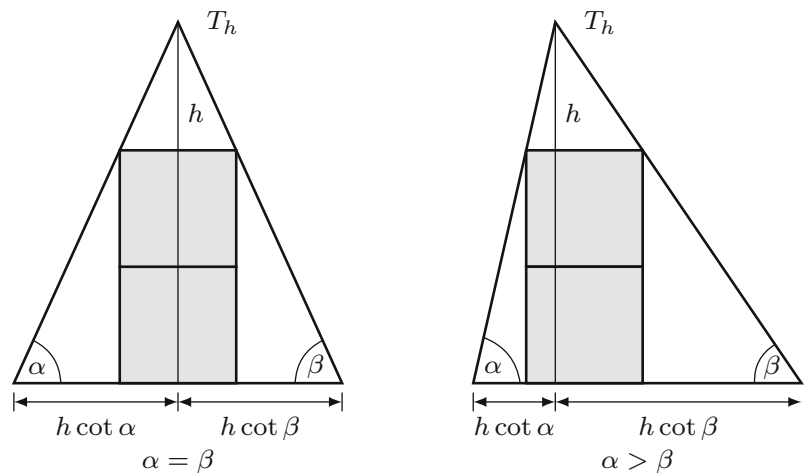

Figure 3. Two congruent squares in $T_{h}$ for $h>1$

Claim 2. Two congruent squares of the side length smaller than or equal to $\max [h /(2 h+1), h /(h+2)]$ can be parallel packed into $T_{h}$ while two squares of the side length greater than $\max [h /(2 h+1), h /(h+2)]$ cannot.

Proof. Let $b$ be the greatest number such that two squares of the side length equal to $b$ can be parallel packed into $T_{h}$ along the base of $T_{h}$ (see Fig. 2). By Thales's intercept theorem, $\frac{1}{2 b}=\frac{h}{h-b}$, i.e., $\quad b=\frac{h}{2 h+1}$. Moreover, let $d$ be the greatest number such that two squares of the side length equal to $d$ can be parallel packed into $T_{h}$ "vertically" as on Fig. 3. By Thales's intercept theorem, $\frac{1}{d}=\frac{h}{h-2 d}$, i.e., $d=\frac{h}{h+2}$.

Corollary 2. Any two congruent squares of the sum of the areas not greater than $\max \left[\frac{4 h}{(2 h+1)^{2}}, \frac{4 h}{(h+2)^{2}}\right] \cdot\left|T_{h}\right|$ can be parallel packed into $T_{h}$. Moreover, $\varrho\left(T_{h}\right) \leq \max \left[\frac{4 h}{(2 h+1)^{2}}, \frac{4 h}{(h+2)^{2}}\right]$.

Proof. If the area of a square equals

$$
\frac{1}{2} \cdot \max \left[\frac{4 h}{(2 h+1)^{2}}, \frac{4 h}{(h+2)^{2}}\right] \cdot\left|T_{h}\right|=\max \left[\frac{h^{2}}{(2 h+1)^{2}}, \frac{h^{2}}{(h+2)^{2}}\right],
$$


then its side length is equal to $\max \left(\frac{h}{2 h+1}, \frac{h}{h+2}\right)$.

It can be check that $4 h /(2 h+1)^{2} \geq 4 h /(h+2)^{2}$ provided $h \leq 1$. Moreover, $2 h /(h+1)^{2} \leq 4 h /(2 h+1)^{2}$ for $h \leq \sqrt{2} / 2$ and $2 h /(h+1)^{2} \leq$ $4 h /(h+2)^{2}$ for $h \geq \sqrt{2}$. Let

$$
\begin{aligned}
\varrho_{h}= & \min \left\{\frac{2 h}{(h+1)^{2}}, \max \left[\frac{4 h}{(2 h+1)^{2}}, \frac{4 h}{(h+2)^{2}}\right]\right\} \\
= & \begin{cases}\frac{2 h}{(h+1)^{2}} & \text { for } 0<h \leq \frac{1}{2} \sqrt{2} \\
\frac{4 h}{(2 h+1)^{2}} & \text { for } \frac{1}{2} \sqrt{2}<h \leq 1 \\
\frac{4 h}{(h+2)^{2}} & \text { for } 1<h \leq \sqrt{2} \\
\frac{2 h}{(h+1)^{2}} & \text { for } \quad h>\sqrt{2}\end{cases}
\end{aligned}
$$

It is easy to verify that $\varrho_{h} \leq 1 / 2$.

Corollary 3. For any $h>0, \varrho\left(T_{h}\right) \leq \varrho_{h}$.

Proof. If either $h \leq \frac{1}{2} \sqrt{2}$ or $h>\sqrt{2}$, then $\varrho_{h}=\frac{2 h}{(h+1)^{2}}$. By Corollary 1 we get $\varrho\left(T_{h}\right) \leq \frac{2 h}{(h+1)^{2}}=\varrho_{h}$. Note that the worst case appears when one square is packed: one square of the area greater than $\varrho_{h}\left|T_{h}\right|$ cannot be packed into $T_{h}$.

If $\frac{1}{2} \sqrt{2}<h \leq 1$, then $\varrho_{h}=\frac{4 h}{(2 h+1)^{2}}=\max \left[\frac{4 h}{(2 h+1)^{2}}, \frac{4 h}{(h+2)^{2}}\right]$. By Corollary 2 we obtain $\varrho\left(T_{h}\right) \leq \varrho_{h}$. Now the worst case appears when two squares are packed: two congruent squares of the total area greater than $\varrho_{h}\left|T_{h}\right|$ cannot be packed either along the base of $T_{h}$ or vertically. However, it is possible to pack two congruent squares of the total area equal to $\varrho_{h}\left|T_{h}\right|$ horizontally (see Fig. 2).

If $1<h \leq \sqrt{2}$, then $\varrho_{h}=\frac{4 h}{(h+2)^{2}}=\max \left[\frac{4 h}{(2 h+1)^{2}}, \frac{4 h}{(h+2)^{2}}\right]$. By Corollary 2 we get $\varrho\left(T_{h}\right) \leq \varrho_{h}$. In that case the worst case appears when two squares are packed: two congruent squares of the total area greater than $\varrho_{h}\left|T_{h}\right|$ cannot be packed either along the base of $T_{h}$ or vertically, but it is possible to pack two congruent squares of the total area equal to $\varrho_{h}\left|T_{h}\right|$ vertically (see Fig. 3).

In Sect. 5 we will show that $\varrho\left(T_{h}\right) \geq \varrho_{h}$.

\section{Lemmas}

Claim 3. Any two squares of the sum of the areas not greater than $\varrho_{h} \cdot\left|T_{h}\right|$ can be parallel packed into $T_{h}$.

Proof. The conditions $\alpha \geq \beta$ and $h \cot \alpha+h \cot \beta=1$ (see Fig. 3) imply that

$$
2 \cot \alpha \leq \cot \alpha+\cot \beta=\frac{1}{h}
$$



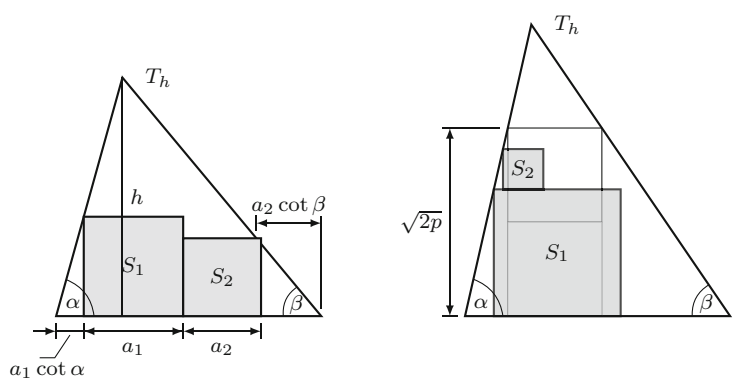

Figure 4. Claim 3

Let $S_{1}$ and $S_{2}$ be the squares of the side lengths $a_{1}, a_{2}$, respectively and let

$$
a_{1}^{2}+a_{2}^{2} \leq \varrho_{h} \cdot\left|T_{h}\right| \leq \max \left[\frac{2 h^{2}}{(2 h+1)^{2}}, \frac{2 h^{2}}{(h+2)^{2}}\right] .
$$

First we show that if $S_{1}$ and $S_{2}$ cannot be packed along the base of $T_{h}$, then $a_{1}^{2}+a_{2}^{2}>\frac{2 h^{2}}{(2 h+1)^{2}}$. If $S_{2}$ cannot be parallel packed into $T_{h}$ "horizontally" as on Fig. 4, left, then $a_{1} \cot \alpha+a_{1}+a_{2}+a_{2} \cot \beta>1$. Since

$$
\begin{aligned}
1 & <a_{1} \cot \alpha+a_{1}+a_{2}+a_{2} \cot \beta=a_{1} \cot \alpha+a_{1}+a_{2}+a_{2}\left(\frac{1}{h}-\cot \alpha\right) \\
& =\left(a_{1}-a_{2}\right) \cot \alpha+a_{1}+a_{2}+a_{2} \cdot \frac{1}{h} \leq \frac{1}{2 h}\left(a_{1}-a_{2}\right)+a_{1}+a_{2}+a_{2} \cdot \frac{1}{h} \\
& =\left(a_{1}+a_{2}\right)\left(1+\frac{1}{2 h}\right)=\frac{2 h+1}{2 h}\left(a_{1}+a_{2}\right),
\end{aligned}
$$

it follows that $a_{1}+a_{2}>\frac{2 h}{2 h+1}$, i.e., $a_{2}>\frac{2 h}{2 h+1}-a_{1}$. Thus

$$
\begin{aligned}
a_{1}^{2}+a_{2}^{2} & >a_{1}^{2}+\left(\frac{2 h}{2 h+1}-a_{1}\right)^{2}=2\left(a_{1}^{2}-\frac{2 h a_{1}}{2 h+1}+\frac{2 h^{2}}{(2 h+1)^{2}}\right) \\
& \geq 2\left(\frac{h^{2}}{(2 h+1)^{2}}-\frac{2 h^{2}}{(2 h+1)^{2}}+\frac{2 h^{2}}{(2 h+1)^{2}}\right)=\frac{2 h^{2}}{(2 h+1)^{2}} .
\end{aligned}
$$

This means that $a_{1}^{2}+a_{2}^{2} \leq \frac{2 h^{2}}{(h+2)^{2}}$. In that case we show that $S_{1}$ and $S_{2}$ can be packed into $T_{h}$ "vertically" as on Fig. 4, right. The assumption $a_{1}^{2} \leq$ $\varrho_{h} \cdot\left|T_{h}\right|$, by Corollary 1 implies that $S_{1}$ can be packed into $T_{h}$. Let $p=a_{1}^{2}+a_{2}^{2}$. Hence $a_{1}+a_{2}=a_{1}+\sqrt{p-a_{1}^{2}}$. The function $f\left(a_{1}\right)=a_{1}+\sqrt{p-a_{1}^{2}}$ has a local maximum at $a_{1}=\sqrt{p / 2}$. This means that $a_{1}+a_{2} \leq \sqrt{p / 2}+\sqrt{p / 2}=\sqrt{2 p}$. Since $a_{1}+a_{2}=\sqrt{2 p}$ for $a_{1}=a_{2}$, it follows that if two congruent squares of the total area $p$ can be parallel packed into $T_{h}$, then any two squares of the total area $p$ can be packed into $T_{h}$. By Corollary 2 two congruent squares of the total area greater than $2 h^{2} /(2 h+1)^{2}$ and not greater than $2 h^{2} /(h+2)^{2}$ can be parallel packed into $T_{h}$ "vertically". 
Let $s T_{h}$ be the image of $T_{h}$ after a homothety with the ratio $s$. Clearly, $s T_{h}$ is a triangle of the base length $s$ and the height $s h$. Since affine transformations preserve relative areas, by Claim 1 and 3 we get the following result.

Corollary 4. Any square of the area not greater than $\varrho_{h} \cdot\left|s T_{h}\right|$ can be parallel packed into $s T_{h}$. Moreover, two squares of the sum of the areas not greater than $\varrho_{h} \cdot\left|s T_{h}\right|$ can be parallel packed into $s T_{h}$.

The following two inequalities will be used in the proof of Lemma 3.

Lemma 1. Let $f(x, h)=\left(1+x^{2}\right) /(2 h+4 h x+x)$. If $h>0$ and if $x \leq 1$, then $f(x, h)>\varrho_{h} /(2 h)$.

Proof. Assume that $h>0$ and that $x \leq 1$. We will show that

$$
f(x, h)>\frac{\varrho_{h}}{2 h}=\left\{\begin{array}{llr}
\frac{1}{(h+1)^{2}} & \text { for } h \in\left(0, \frac{1}{2} \sqrt{2}\right] \cup(\sqrt{2},+\infty) \\
\frac{2}{(2 h+1)^{2}} & \text { for } & h \in\left(\frac{1}{2} \sqrt{2}, 1\right] . \\
\frac{2}{(h+2)^{2}} & \text { for } & h \in(1, \sqrt{2}]
\end{array} .\right.
$$

Observe that

$$
\begin{aligned}
f(x, h)-\frac{1}{(h+1)^{2}} & =\frac{x^{2} h^{2}+2 x^{2} h+x^{2}-4 h x-x+h^{2}+1}{(2 h+4 h x+x)(h+1)^{2}} \\
& =\frac{(h x-1)^{2}(1-x)+(x-h)^{2}+x^{3} h^{2}}{(2 h+4 h x+x)(h+1)^{2}}>0
\end{aligned}
$$

for $h>0$. Moreover, if $h \geq \sqrt{2} / 2$, then $1 /(h+1)^{2} \geq 2 /(2 h+1)^{2}$ and, consequently, $f(x, h)>\frac{2}{(2 h+1)^{2}}$. Finally observe that

$$
\begin{aligned}
f(x, h)-\frac{2}{(h+2)^{2}} & =\frac{x^{2} h^{2}+4 x^{2} h+4 x^{2}-8 h x-2 x+h^{2}+4}{(2 h+4 x h+x)(h+2)^{2}} \\
& =\frac{(h x-2)^{2}(1-x)+(2 x-h)^{2}+x^{3} h^{2}+2 x}{(2 h+4 x h+x)(h+2)^{2}}>0 .
\end{aligned}
$$

Lemma 2. Let $g(y, h)=(2+y) /(6 h+4 h y+1)$. If $h>0$ and if $y>0$, then $g(y, h)>\varrho_{h} /(2 h)$.

Proof. We proceed as in the proof of Lemma 1. Observe that

$$
g(y, h)-\frac{1}{(h+1)^{2}}=\frac{h^{2}+(y+1)(h-1)^{2}}{(6 h+4 h y+1)(h+1)^{2}}>0
$$

and

$$
g(y, h)-\frac{2}{(h+2)^{2}}=\frac{4+2(h-1)^{2}+y(h-2)^{2}}{(6 h+4 h y+1)(h+2)^{2}}>0 .
$$



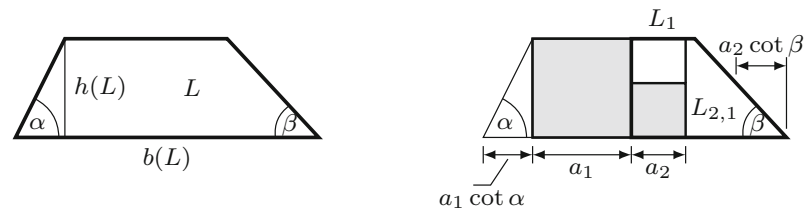

Figure 5. Trapezoids $L$ and $L_{1}$

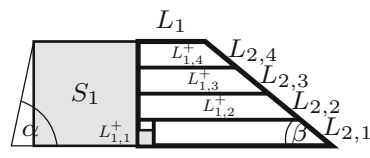

$m_{2}=2, \quad L_{2,1} \subset L_{1,1}^{+}$

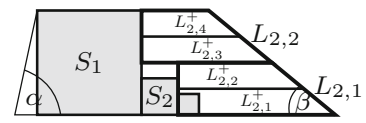

$m_{2}=1, k=3$ and $n_{k}=1$

Figure 6. Division of $L_{1}$

\section{Packing Squares into Trapezoids}

In the main packing method squares will be packed in trapezoid-shape layers. In this section an algorithm for packing squares into trapezoids is described.

Assume that $S_{i}$ is a square of the side length $a_{i}$, where $a_{1} \geq a_{2} \geq \ldots$ Let $L$ be a trapezoid of the base length $b(L)$, with the height $h(L)$ and with the base angles measuring $\alpha$ and $\beta$, where $90^{\circ} \geq \alpha \geq \beta$ (see Fig. 5, left). Moreover assume that $a_{1}(1+\cot \alpha)+a_{2}(1+\cot \beta) \leq b(L)$ and that $h(L)=a_{1}$ (see Fig. 5, right).

\section{Description of the $L$-method for packing $S_{1}, S_{2}, \ldots$ into $L$.}

- The first square $S_{1}$ is packed into $L$ as far to the left as possible. Let $L_{1}$ be the part of $L$ lying to the right of $S_{1}$, i.e., the trapezoid of the height $h(L)=a_{1}$, of the base length $b\left(L_{1}\right)=b(L)-a_{1}(1+\cot \alpha)$ and with the base angles measuring $90^{\circ}$ and $\beta$ (see Fig. 5 , right).

- If $a_{2}>\frac{1}{2} a_{1}$ (see Fig. 5), then $S_{2}$ is packed into $L_{1}$ in the left-bottom corner; then $L_{2,1}$ is the part of $L_{1}$ lying to the right of $S_{2}$, i.e., either $L_{2,1}$ is the trapezoid of the height $h(L)=a_{1}$, of the base length $b\left(L_{1}\right)=b(L)-$ $a_{1}(1+\cot \alpha)-a_{2}$ and with the base angles measuring $90^{\circ}$ and $\beta$ or $L_{2,1}$ is the triangle of the base length $b\left(L_{1}\right)=b(L)-a_{1}(1+\cot \alpha)-a_{2}$, with the base angles measuring $90^{\circ}$ and $\beta$, and of the height not greater than $h(L)=a_{1}$. If $a_{2} \leq \frac{1}{2} a_{1}$, then $L_{1}$ is partitioned into smaller trapezoids. Let $m_{2}$ be an integer such that $2^{-m_{2}-1} a_{1}<a_{2} \leq 2^{-m_{2}} a_{1}$. Then $L_{1}$ is divided into $2^{m_{2}}$ trapezoids $L_{1,1}^{+}, \ldots, L_{1,2^{m_{2}}}^{+}$of the height $h_{2}=2^{-m_{2}} a_{1}$ (see Fig. 6). The square $S_{2}$ is packed into $L_{1,1}^{+}$as far to the left as possible, i.e., in the left-bottom corner. New trapezoids are defined as follows: 
$L_{2,1}$ is the part of $L_{1,1}^{+}$lying to the right of $S_{2}$ and $L_{2, i}=L_{1, i}^{+}$for $i=2,3, \ldots, 2^{m_{2}}$ (it is possible that $L_{2,1}$ is a triangle).

- Assume that squares $S_{1}, \ldots, S_{k-1}$ were packed and that trapezoids $L_{k-1, i}$ for $i=1,2, \ldots, 2^{m_{k-1}}$ (of the height $h_{k-1}$ ) were defined. Next square $S_{k}$ is placed in the following way. If $a_{k}>\frac{1}{2} h_{k-1}$, then let $j$ be the smallest integer such that $b\left(L_{k-1, j}\right) \geq a_{k}(1+\cot \beta)$ (we find the lowest lying trapezoid into which $S_{k}$ can be packed). Then $S_{k}$ is packed into $L_{k-1, j}$ in the left-bottom corner. New trapezoids are defined: $L_{k, j}$ is the part of $L_{k-1, j}$ lying to the right of $S_{k}$ and $L_{k, i}=$ $L_{k-1, i}$ for $i \neq j$ (it is possible that some trapezoids are triangles). For example, squares $S_{2}, S_{3}$ and $S_{4}$ are packed on Fig. 8 (left) by this rule. Moreover, squares $S_{4}, \ldots, S_{10}$ are packed by this rule on Fig. 9 , left. If $a_{k} \leq \frac{1}{2} h_{k-1}$, then each trapezoid $L_{k-1, i}$ is partitioned into smaller trapezoids. Let $n_{k}$ be an integer such that $2^{-n_{k}-1} h_{k-1}<a_{k} \leq$ $2^{-n_{k}} h_{k-1}$. Then $L_{k-1, i}$ (for $i=1, \ldots, 2^{m_{k-1}}$ ) is divided into $2^{n_{k}}$ trapezoids $L_{k-1,(i-1) 2^{n_{k}+1}}^{+}, \ldots, L_{k-1, i 2^{n_{k}}}^{+}$of the height $2^{-n_{k}} h_{k-1}$ (see Fig. 6, right). Let $j$ be the smallest integer such that $b\left(L_{k-1, j}^{+}\right) \geq a_{k}(1+\cot \beta)$ (we find the lowest lying trapezoid into which $S_{k}$ can be packed). The square $S_{k}$ is packed into $L_{k-1, j}^{+}$in the left-bottom corner. New trapezoids are defined: $L_{k, j}$ is the part of $L_{k-1, j}^{+}$lying to the right of $S_{k}$ and $L_{k, i}=L_{k-1, i}^{+}$for $i \neq j$ and $i \in\left\{1,2, \ldots, 2^{m_{k}}\right\}$, where $m_{k}=m_{k-1} n_{k}$ (it is possible that some trapezoids are triangles). For example, squares $S_{3}$ and $S_{9}$ are packed on Fig. 9 (right) by this rule.

Lemma 3. Assume that $S_{i}$ is a square of the side length $a_{i}$ for $i=1,2, \ldots$, where $a_{1} \geq a_{2} \geq \ldots$. Let $L$ be a trapezoid of the base length $b(L)$, with the height $h(L)=a_{1}$ and with the base angles measuring $\alpha$ and $\beta$ (see Fig. 5, left). Moreover assume that $a_{1}(1+\cot \alpha)+a_{2}(1+\cot \beta) \leq b(L)$. If $S_{z}$ is the first square that cannot be packed into $L$ by the $L$-method, then $\left|S_{1}\right|+\ldots+\left|S_{z-1}\right|>$ $\varrho_{h} \cdot|L|$.

Proof. Since any homothety preserves relative areas, without loss of generality one can assume that $b(L)=1$.

Consider four cases.

Case 1: $z=3, a_{z} \geq a_{1} / 2$. (see Fig. 7 )

Let $x=a_{z} / a_{1}$. Since $a_{z}$ cannot be packed into $L$, it follows that $a_{1} \cot \alpha+$ $a_{1}+a_{2}+a_{z}+a_{z} \cot \beta>1$. Recall that $\cot \alpha+\cot \beta=1 / h$ and $\cot \alpha \leq \frac{1}{2 h}$. By

$$
\begin{aligned}
1 & <a_{1} \cot \alpha+a_{1}+a_{2}+a_{z}+a_{z} \cot \beta=a_{1} \cot \alpha+a_{1}+2 a_{z}+a_{z}\left(\frac{1}{h}-\cot \alpha\right) \\
& =\left(a_{1}-a_{z}\right) \cot \alpha+a_{1}+2 a_{z}+a_{z} \cdot \frac{1}{h} \leq \frac{1}{2 h}\left(a_{1}-a_{1} x\right)+a_{1}+2 a_{1} x+a_{1} x \cdot \frac{1}{h} \\
& =a_{1}\left(\frac{1+x}{2 h}+1+2 x\right)=a_{1} \cdot \frac{1+x+4 h x+2 h}{2 h}
\end{aligned}
$$



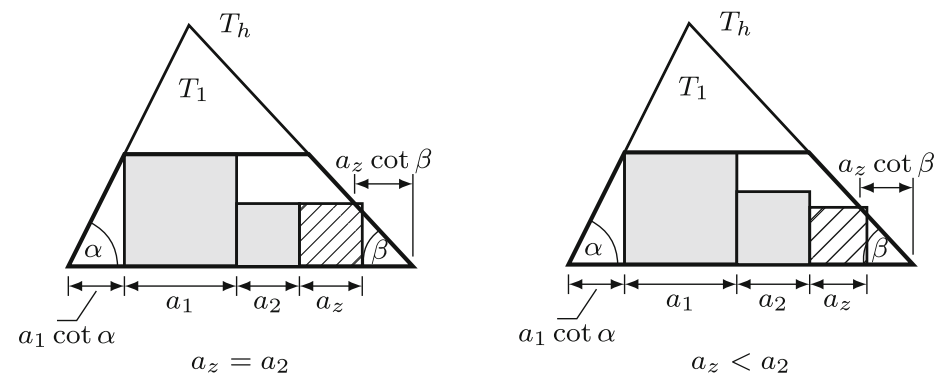

FiguRe $7 . z=3, a_{z} \geq a_{1} / 2$

we get

$$
a_{1}>\frac{2 h}{1+2 h+4 h x+x} .
$$

Denote by $\varrho(L)$ the ratio of the sum of the areas of squares packed into $L$ to the area of $L$. Only two squares are packed in $L$, therefore

$$
\varrho(L)=\frac{a_{1}^{2}+a_{2}^{2}}{a_{1}-\frac{1}{2} a_{1}^{2}(\cot \alpha+\cot \beta)}=\frac{a_{1}^{2}+\left(x a_{1}\right)^{2}}{a_{1}-\frac{a_{1}^{2}}{2 h}}=\frac{a_{1}\left(1+x^{2}\right) \cdot 2 h}{2 h-a_{1}} .
$$

Since the function $f\left(a_{1}\right)=2 h a_{1}\left(1+x^{2}\right) /\left(2 h-a_{1}\right)$ is increasing, it follows that

$$
\varrho(L)>\frac{2 h\left(1+x^{2}\right) \cdot 2 h}{1+2 h+4 h x+x} \cdot \frac{1+2 h+4 h x+x}{2 h \cdot(2 h+4 h x+x)}=2 h \frac{\left(1+x^{2}\right)}{2 h+4 h x+x} .
$$

By Lemma 1, $\varrho(L)>2 h \cdot \varrho_{h} /(2 h)=\varrho_{h}$.

Case 2: $z>3, a_{z} \geq a_{1} / 2$.

Let $L^{-}$be the trapezoid with $b\left(L^{-}\right)=1-a_{3}-\ldots-a_{z-1}$, with the height $a_{1}$ and the base angles with the same measures as the base angles of $L$ (see Fig. 8, where $z=5$ ). Moreover, let $s L^{-}$be the image of $L^{-}$after a homothety with the ratio $s$ such that the larger base of $s L^{-}$is equal to 1 . By Case 1 , the density of packing squares $s S_{1}$ and $s S_{2}$ into $s L^{-}$is greater than $\varrho_{h}$. This implies that the density of packing squares $S_{1}$ and $S_{2}$ into $L^{-}$is greater than $\varrho_{h}$. The density of packing squares $S_{3}, \ldots, S_{z-1}$ into the rectangle $R_{L}=\left(a_{3}+\ldots+a_{z-1}\right) \times a_{1}$ is greater than $1 / 2 \geq \varrho_{h}$. Clearly, $\left|L^{-}\right|+\left|R_{L}\right|=|L|$. As a consequence, the density of packing squares $S_{1}, S_{2}, \ldots, S_{z-1}$ into $L$ is greater than $\varrho_{h}$.

Case 3: $z \geq 3, a_{1} / 4<a_{z} \leq a_{1} / 2$.

Let $k$ be the smallest integer such that $a_{k}<a_{1} / 2$ (see Fig. 9, left). Obviously, $k \leq z$. Let $L_{k-1,1}$ be the part of $L_{1}$ lying to the right of $S_{k-1}$, i.e., either the trapezoid of the height $h_{r}=h\left(L_{k-1,1}\right)$, of the base length $b_{r}=b\left(L_{k-1, i}\right)$ and with the base angles measuring $90^{\circ}$ and $\beta$ or the triangle 

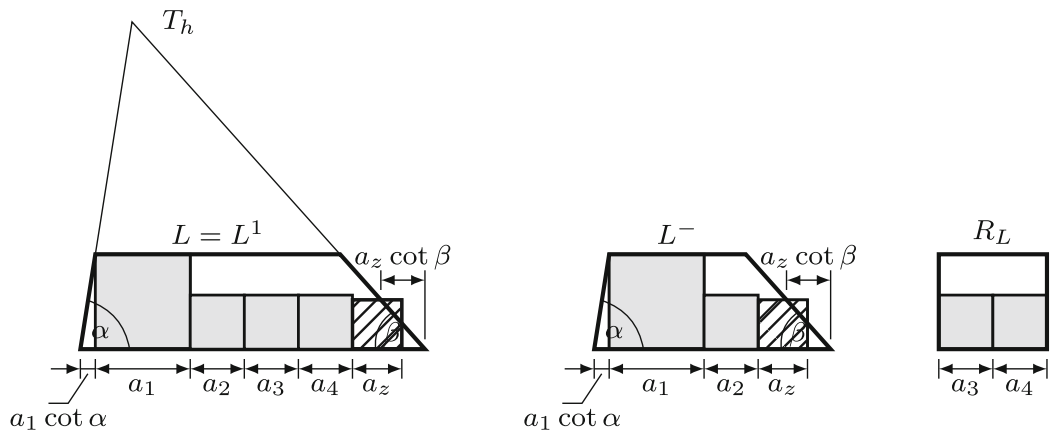

FiguRE $8 . z=5, a_{z}>a_{1} / 2$

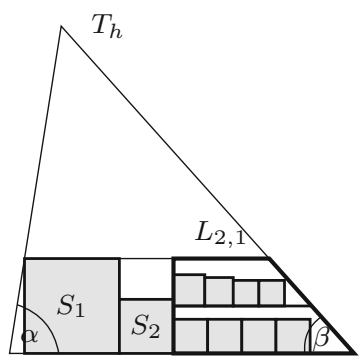

$a_{1} / 4<a_{z} \leq a_{1} / 2$

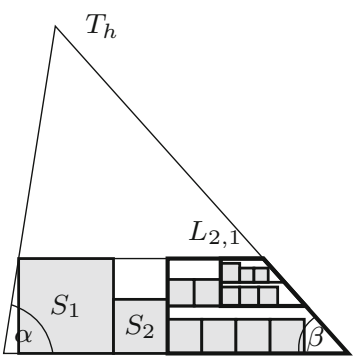

$a_{z} \leq a_{1} / 4$

FiguRE $9 . \quad k=3$

of the base length $b_{r}=b\left(L_{k-1,1}\right)$, with the base angles measuring $90^{\circ}$ and $\beta$, and of the the height not greater than $h_{r}=h\left(L_{k-1,1}\right)$,

Squares $S_{k}, S_{k+1}, \ldots$ are packed into two rectangular trapezoids $L_{k-1,1}^{+}$ and $L_{k-1,2}^{+}$with disjoint interiors, where $L_{k-1,1}=L_{k-1,1}^{+} \cup L_{k-1,2}^{+}$(it is possible that trapezoids are triangles).

Assume that $S_{v}$ is the first square that cannot be packed into $L_{k-1,1}^{+}$. Clearly $a_{v} \leq h_{r} / 2$ and $a_{z} \leq h_{r} / 2$ (see Fig. 10, right).

Let $p_{2}=\left|S_{k}\right|+\ldots+\left|S_{z-1}\right|$, i.e., $p_{2}$ is equal to the sum of the areas of squares packed into $L_{k-1,1}$.

Subcase 3A: $b_{r} \geq a_{v}(1+\cot \beta)+\frac{1}{2} h_{r} \cot \beta$. Observe that (see Fig. 10, right)

$$
p_{2} \geq\left[b_{r}-a_{v}(1+\cot \beta)\right] a_{v}+\left[b_{r}-a_{z}(1+\cot \beta)-\frac{1}{2} h_{r} \cot \beta\right] a_{z} .
$$

Let

$$
p_{1}=\left[b_{r}-\frac{1}{2} h_{r}(1+\cot \beta)\right] \frac{1}{2} h_{r}
$$



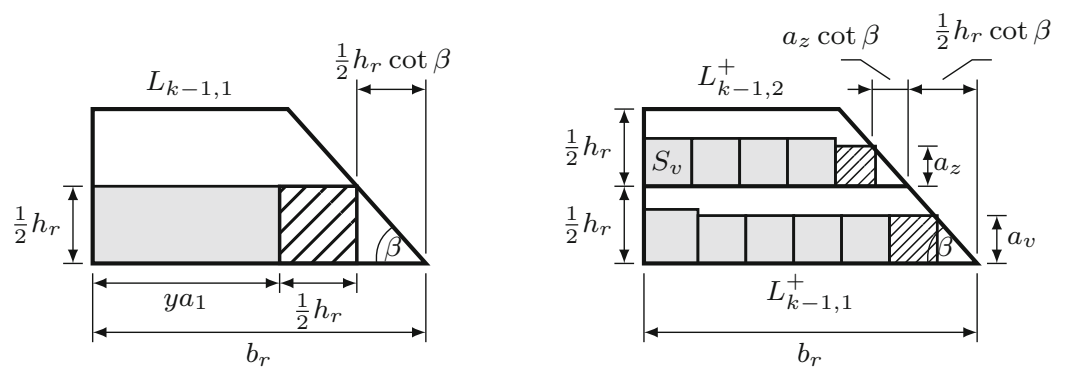

Figure 10. Subcase 3A

and let $y=\left(2 p_{1}\right) /\left(a_{1} h_{r}\right)$. Obviously, $p_{1}$ is equal to the area of the gray rectangle and $y a_{1}$ is equal to the base length of the gray rectangle on Fig. 10, left.

Since

$$
\begin{aligned}
p_{2}-p_{1} \geq & b_{r}\left(a_{v}+a_{z}-\frac{1}{2} h_{r}\right)+(1+\cot \beta)\left(\frac{1}{4} h_{r}^{2}-a_{v}^{2}-a_{z}^{2}\right)-\frac{1}{2} h_{r} a_{z} \cot \beta \\
> & {\left[a_{v}(1+\cot \beta)+\frac{1}{2} h_{r} \cot \beta\right]\left(a_{v}+a_{z}-\frac{1}{2} h_{r}\right) } \\
& +(1+\cot \beta)\left(\frac{1}{4} h_{r}^{2}-a_{v}^{2}-a_{z}^{2}\right)-\frac{1}{2} h_{r} a_{z} \cot \beta \\
= & a_{z}(1+\cot \beta)\left(a_{v}-a_{z}\right)+\frac{1}{2} h_{r}\left(\frac{h_{r}}{2}-a_{v}\right)
\end{aligned}
$$

and $a_{z} \leq a_{v} \leq h_{r} / 2$, we get $p_{2}>p_{1}$. Hence, to show that $\sum_{i=1}^{z-1}\left|S_{i}\right|>\varrho_{h}|L|$, it suffices to verify that

$$
a_{1}^{2}+\ldots+a_{k-1}^{2}+p_{1}>\varrho_{h}|L| .
$$

First assume that $k=2$. We argue as in Case 1 . By conditions $a_{1} \cot \alpha+$ $a_{1}+y a_{1}+\frac{1}{2} a_{1}+\frac{1}{2} a_{1} \cot \beta=1, \cot \alpha+\cot \beta=1 / h$ and $\cot \alpha \leq 1 /(2 h)$, we get

$$
\begin{aligned}
1 & =a_{1} \cot \alpha+a_{1}+y a_{1}+\frac{1}{2} a_{1}+\frac{1}{2} a_{1} \cot \beta \\
& =a_{1} \cot \alpha+\frac{3}{2} a_{1}+y a_{1}+\frac{1}{2} a_{1}\left(\frac{1}{h}-\cot \alpha\right) \\
& =\frac{1}{2} a_{1} \cot \alpha+\frac{3}{2} a_{1}+y a_{1}+\frac{a_{1}}{2 h} \\
& \leq \frac{1}{2} a_{1} \cdot \frac{1}{2 h}+\frac{3}{2} a_{1}+y a_{1}+\frac{a_{1}}{2 h}=a_{1} \cdot \frac{3 h+2 y h+\frac{3}{2}}{2 h} .
\end{aligned}
$$

Consequently,

$$
a_{1} \geq \frac{4 h}{6 h+4 y h+3}
$$



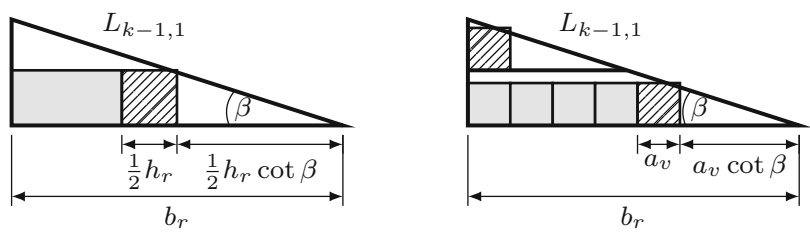

Figure 11. Subcase 3C

Now we estimate the packing density:

$$
\begin{aligned}
\frac{a_{1}^{2}+p_{1}}{|L|} & =\frac{a_{1}^{2}+\frac{1}{2} a_{1} \cdot y a_{1}}{a_{1}-\frac{a_{1}^{2}}{2 h}}=h(2+y) \cdot \frac{a_{1}}{2 h-a_{1}} \\
& \geq h(2+y) \cdot \frac{\frac{4 h}{6 h+4 y h+3}}{2 h-\frac{4 h}{6 h+4 y h+3}}=2 h \cdot \frac{2+y}{6 h+4 h y+1} .
\end{aligned}
$$

By Lemma 2, $a_{1}^{2}+p_{1}>|L| \cdot 2 h \cdot \varrho_{h} /(2 h)=\varrho_{h} \cdot|L|$.

If $k>2$, then we argue as in Case 2 .

Subcase 3B: $b_{r}<\frac{1}{2} h_{r}(1+\cot \beta)$. This assumption implies that a square of the side length greater than $h_{r} / 2$ cannot be packed in $L_{k-1,1}$. By Case 1 and Case $2, a_{1}^{2}+\ldots+a_{k-1}^{2}>\varrho_{h}|L|$.

Subcase $3 C: \frac{1}{2} h_{r}(1+\cot \beta) \leq b_{r} \leq a_{v}(1+\cot \beta)+\frac{1}{2} h_{r} \cot \beta$. The total area of squares packed in two trapezoids contained in $L_{k-1,1}$ (see Fig. 11, right) is not smaller than

$$
p_{2}^{-}=\left[b_{r}-a_{v}(1+\cot \beta)\right] a_{v} .
$$

In this subcase, similarly as in Subcase $3 \mathrm{a}$, it suffices to check that $p_{2}^{-}\left(L_{k-1,1}\right)$ $\geq p_{1}^{-}\left(L_{k-1,1}\right)$, where

$$
p_{1}^{-}=\left[b_{r}-\frac{1}{2} h_{r}(1+\cot \beta)\right] \cdot \frac{1}{2} h_{r}
$$

( $p_{1}^{-}$is equal to the area of the gray rectangle on Fig. 11, left). Observe that

$$
\begin{aligned}
p_{2}^{-}-p_{1}^{-} & =b_{r} a_{v}-a_{v}^{2}(1+\cot \beta)-\frac{1}{2} h_{r} b_{r}+\frac{1}{4} h_{r}^{2}(1+\cot \beta) \\
& =\left(\frac{1}{4} h_{r}^{2}-a_{v}^{2}\right)(1+\cot \beta)+b_{r} a_{v}-\frac{1}{2} b_{r} h_{r} \\
& =\left(\frac{1}{2} h_{r}-a_{v}\right)\left(\frac{1}{2} h_{r}+a_{v}\right)(1+\cot \beta)+\left(\frac{1}{2} h_{r}-a_{v}\right) b_{r} \\
& =\left(\frac{1}{2} h_{r}-a_{v}\right)\left[\frac{1}{2} h_{r} \cot \beta+a_{v}(1+\cot \beta)-b_{r}+\frac{1}{2} h_{r}\right] .
\end{aligned}
$$

By $\frac{1}{2} h_{r}-a_{v} \geq 0$ and $\frac{1}{2} h_{r} \cot \beta+a_{v}(1+\cot \beta)-b_{r}>0$, we get $p_{2}^{-}-p_{1}^{-}>0$.

Case 4 : $z \geq 3, a_{z} \leq a_{1} / 4$ (see Fig. 9, right). 
We proceed in the same way as in Case 3. The sum of the areas of squares packed into two trapezoids $L_{l, i}^{+}, L_{l, i+1}^{+}$contained in the trapezoid $L_{l, i}^{+} \cup L_{l, i+1}^{+}$ is greater than the area of the corresponding "gray" rectangle of the height $h\left(L_{l, i}^{+}\right)$and the base length $b\left(L_{l, i}^{+}\right)-h\left(L_{l, i}^{+}\right) \cdot(1+\cot \beta)$ contained in $L_{l, i}^{+} \cup$ $L_{l, i+1}^{+}$.

\section{Main Result}

Theorem 1. Any collection of squares of the total area not greater than $\varrho_{h} \cdot\left|T_{h}\right|$ can be parallel packed into $T_{h}$.

Proof. Let $S_{1}, S_{2}, \ldots$ be a collection of squares of the total area not greater than $\varrho_{h} \cdot\left|T_{h}\right|$. There is no loss of generality in assuming that $a_{1} \geq a_{2} \geq \ldots$, where $a_{i}$ denotes the side length of $S_{i}$.

Squares will be packed into trapezoid-shape layers in such a way that the packing density in each layer, i.e., the ratio of the sum of packed squares to the area of the layer, is greater than $\varrho_{h}$.

Since $a_{1}^{2}+a_{2}^{2}+\ldots \leq \varrho_{h} \cdot\left|T_{h}\right|$, by Corollary 1 the first square can be packed into $T_{h}$. Moreover, if there are at least two squares in the collection (i.e., if $z>1$ ), then, by Claim 3 , squares $S_{1}$ and $S_{2}$ can be packed into $T_{h}$ either horizontally or vertically, i.e., either $a_{1}(1+\cot \alpha)+a_{2}(1+\cot \beta) \leq 1$ or $a_{1}+a_{2} \leq 2 h /(h+2)$ (see the proof of Claim 3 ).

If $a_{1}(1+\cot \alpha)+a_{2}(1+\cot \beta) \leq 1$ (see Fig. 13), then let $L^{1}$ be the trapezoid contained in $T_{h}$ with the base length 1 , the height $a_{1}$ and the base angles measuring $\alpha$ and $\beta$. This trapezoid is called the basic layer. Squares $S_{1}, S_{2}, \ldots$ are packed into $L^{1}$ by the $L$-method. Denote by $S_{n_{1}}$ the first square that cannot be packed into $L^{1}\left(n_{1}=3\right.$ on Fig. 13). By Lemma 3 , the total area of squares packed into $L^{1}$ is not smaller than $\varrho_{h} \cdot\left|L^{1}\right|$.

If $a_{1}(1+\cot \alpha)+a_{2}(1+\cot \beta)>1$ and at the same time $a_{1}+a_{2} \leq \frac{2 h}{2+h}$ (this case is possible for $h>1$ ), then we pack $S_{1}$ and $S_{2}$ "vertically". The first condition implies that $1<\left(a_{1}+a_{2}\right)(2 h+1) /(2 h)$ (see the proof of Claim $3)$. Let $\lambda=a_{1}+a_{2}$. The so-called double layer $L^{1}$ of the height $\lambda$ is created for packing only two squares, see Fig. 12 . In that case $n_{1}=3\left(S_{n_{1}}\right.$ is the first square that is not packed into $\left.L^{1}\right)$ and $\lambda>2 h /(2 h+1)$. It is easy to verify that $a_{1}^{2}+a_{2}^{2} \geq(\lambda / 2)^{2}+(\lambda / 2)^{2}=\lambda^{2} / 2$. Recall that $\varrho_{h} \leq 1 / 2$ while the packing density in the double layer is not smaller than $1 / 2$ :

$$
\frac{a_{1}^{2}+a_{2}^{2}}{1 \cdot \lambda-\frac{\lambda^{2}}{2 h}} \geq \frac{\frac{1}{2} \lambda^{2}}{\lambda-\frac{\lambda^{2}}{2 h}}=\frac{h \lambda}{2 h-\lambda}>\frac{h \cdot \frac{2 h}{2 h+1}}{2 h-\frac{2 h}{2 h+1}}=\frac{1}{2} .
$$

Since the sum of the areas of the squares packed into $L^{1}$ is greater than $\varrho_{h} \cdot\left|L^{1}\right|$, it follows that the total area of the remaining squares is smaller than $\varrho_{h} \cdot\left|T_{1}\right|$, where $T_{1}=T_{h} \backslash L^{1}$.

For packing squares $S_{n_{1}}, S_{n_{1}+1}, \ldots$ the new layer $L^{2}$ of the base length $1-a_{1} \cot \alpha-a_{1} \cot \beta$, with the base angles measuring $\alpha$ and $\beta$ and with the 


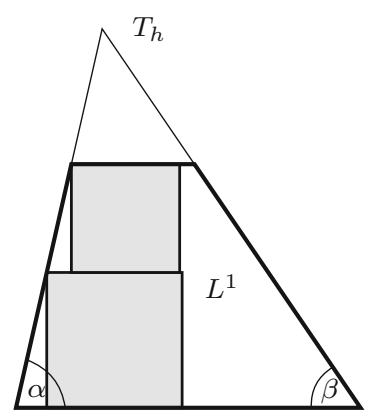

FiguRE 12. $a_{1}+a_{2}>2 h /(2 h+1)$

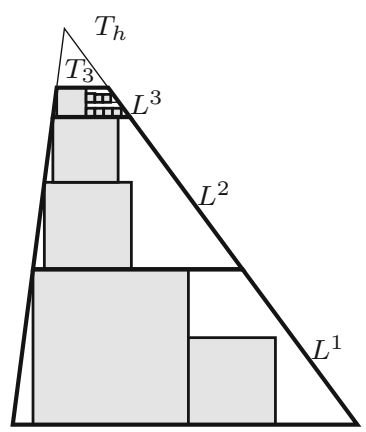

Figure 13. Packing method

height either $a_{n_{1}}$ or $a_{n_{1}}+a_{n_{1}+1}$ is created directly above $L^{1}$ (see Fig. 13, where $L^{2}$ is a double layer). If $a_{n_{1}}(1+\cot \alpha)+a_{n_{1}+1}(1+\cot \beta) \leq b\left(L^{2}\right)$, then we place $S_{n_{1}}, S_{n_{1}+1}, \ldots$ into the basic layer $L^{2}$ of the height $a_{n_{1}}$ by the $L$-method (denote by $S_{n_{2}}$ the first square that cannot be packed into $L^{2}$ ). Otherwise, we pack $S_{n_{1}}$ and $S_{n_{1}+1}$ into the double layer of the height $a_{n_{1}}+a_{n_{1}+1}$ and we take $n_{2}=n_{1}+2$.

Assume that the layers $L^{1}, \ldots, L^{p}$ were created and that $S_{n_{p}}$ is the first square that cannot be packed into $L^{p}$. The total area of squares packed into each $L^{i}(i=1, \ldots, p)$ is greater than $\varrho_{p} \cdot\left|L^{i}\right|$. This implies that $a_{n_{p}+1}^{2}+a_{n_{p}+2}^{2}+$ $\ldots$ is smaller than $\varrho_{h}$ times the area of $T_{p}=T_{h} \backslash \bigcup_{i=1}^{p} L^{i}$. By Corollary $4, S_{n_{p}}$ can be packed into $T_{p}$. Moreover by Claim $3, S_{n_{p}}$ and $S_{n_{p}+1}$ can be packed into $T_{p}$ provided $z \geq n_{p}+1$. There is an empty space in $T_{h}$ to create either the basic or the double layer to pack $S_{n_{p}}$ and $S_{n_{p}+1}$. This means that all the squares can be packed into $T_{h}$.

The following result is a consequence of Theorem 1 and Corollary 3. 
Corollary 5. For any $h>0, \varrho\left(T_{h}\right)=\varrho_{h}$.

Funding This research was partially supported by National Natural Science Foundation of China (11471095), the NSF of Hebei Province (A2021205008), and the Science Foundation of Hebei Normal University (L2020Z01).

Availability of data and material Not applicable.

\section{Declarations}

Conflict of interest The authors declare that they have no conflict of interest.

Code availability Not applicable.

Ethics approval Not applicable.

Consent to participate Not applicable.

Consent for publication Not applicable.

Open Access. This article is licensed under a Creative Commons Attribution 4.0 International License, which permits use, sharing, adaptation, distribution and reproduction in any medium or format, as long as you give appropriate credit to the original author(s) and the source, provide a link to the Creative Commons licence, and indicate if changes were made. The images or other third party material in this article are included in the article's Creative Commons licence, unless indicated otherwise in a credit line to the material. If material is not included in the article's Creative Commons licence and your intended use is not permitted by statutory regulation or exceeds the permitted use, you will need to obtain permission directly from the copyright holder. To view a copy of this licence, visit http://creativecommons. org/licenses/by/4.0/.

\section{References}

[1] Bálint, V.: A packing problem and geometrical series. In: Fourth Czechoslovakian Symposium on Combinatorics, Graphs and Complexity, vol. 51 of Annals of Discrete Mathematics, pp. 17-21. Elsevier (1992)

[2] Chalcraft, A.: Perfect square packings. J. Comb. Theory Ser. A 92, 158-172 (2000)

[3] Erdös, P., Graham, R.L.: On packing squares with equal squares. J. Comb. Theory Ser. A 19(1), 119-123 (1975)

[4] Fu, M., Lian, Y., Zhang, Y.: On parallel packing and covering of squares and cubes. Results Math. 74(4), 1-13 (2019)

[5] Grzegorek, P., Januszewski, J.: A note on three Moser's problems and two Paulhus' lemmas. J. Comb. Theory Ser. A 162, 222-230 (2019) 
[6] Januszewski, J.: Parallel packing and covering of an equilateral triangle with sequences of squares. Acta Math. Hung. 125(3), 249-260 (2009)

[7] Januszewski, J.: Packing rectangles into a large square. Period. Math. Hung. 72(1), 90-101 (2016)

[8] Januszewski, J., Zielonka, Ł: A note on perfect packing of squares and cubes. Acta Math. Hung. 163(2), 530-537 (2021)

[9] Joós, A.: On packing of rectangles in a rectangle. Discrete Math. 341(9), 2544$2552(2018)$

[10] Joós, A.: Perfect square packings. Math. Rep., accepted

[11] Moon, J.W., Moser, L.: Some packing and covering theorems. Colloq. Math. 17, 103-110 (1967)

[12] Paulhus, M.M.: An algorithm for packing squares. J. Comb. Theory Ser. A 82(2), 147-157 (1998)

[13] Su, Z., Lu, M., Liu, X.: Parallel packing of triangles with squares. Rocky Mountain J. Math., accepted. https://projecteuclid.org/journals/ rmjm/rocky-mountain-journal-of-mathematics/DownloadAcceptedPapers/ 210129-ZhanjunSu.pdf (2021). Accessed 16 Aug 2021

Janusz Januszewski and Łukasz Zielonka

Institute of Mathematics and Physics

Bydgoszcz University of Science and Technology

Al. Prof. S. Kaliskiego 7

85-789 Bydgoszcz

Poland

e-mail: januszew@pbs.edu.pl;

Lukasz.Zielonka@pbs.edu.pl

$\mathrm{Xi}$ Liu and Zhanjun Su

School of Mathematical Sciences

Hebei Normal University

Shijiazhuang 050024

China

e-mail: $1298789840 @ q q . c o m ;$

suzj888@163.com

Received: August 16, 2021.

Accepted: December 20, 2021.

Publisher's Note Springer Nature remains neutral with regard to jurisdictional claims in published maps and institutional affiliations. 